\title{
BMJ Open Reproductive patterns, pregnancy outcomes and parental leave practices of women physicians in Ontario, Canada: the Dr Mom Cohort Study protocol
}

\author{
Maria C Cusimano (D) , ,2 Nancy N Baxter, ${ }^{2,3,4}$ Rinku Sutradhar, ${ }^{4,5}$ Joel G Ray, ${ }^{4,6}$ \\ Amit X Garg, ${ }^{4,7}$ Eric McArthur, ${ }^{4}$ Simone Vigod,,${ }^{4,8,9}$ Andrea N Simpson ${ }^{1,2,4,10}$
}

To cite: Cusimano MC, Baxter NN, Sutradhar R, et al. Reproductive patterns, pregnancy outcomes and parental leave practices of women physicians in Ontario, Canada: the Dr Mom Cohort Study protocol. BMJ Open 2020;10:e041281. doi:10.1136/ bmjopen-2020-041281

- Prepublication history and additional material for this paper are available online. To view these files, please visit the journal online (http://dx.doi org/10.1136/bmjopen-2020041281).

Received 03 June 2020 Revised 18 August 2020 Accepted 16 September 2020

Check for updates

(C) Author(s) (or their employer(s)) 2020. Re-use permitted under CC BY-NC. No commercial re-use. See rights and permissions. Published by BMJ.

For numbered affiliations see end of article.

Correspondence to

Dr Andrea N Simpson;

Andrea.Simpson@unityhealth.to

\section{ABSTRACT}

Introduction Surveys and qualitative studies suggest that women physicians may delay childbearing, be at increased risk of adverse peripartum complications when they do become pregnant, and face discrimination and lower earnings as a result of parenthood. Observational studies enrolling large, representative samples of women physicians are needed to accurately evaluate their reproductive patterns, pregnancy outcomes, parental leave practices and earnings. This protocol provides a detailed research plan for such studies.

Methods and analysis The Dr Mom Cohort Study encompasses a series of retrospective observational studies of women physicians in Ontario, Canada. All practising physicians in Ontario are registered with the College of Physicians and Surgeons of Ontario (CPSO). By linking a dataset of physicians from the CPSO to existing provincial administrative databases, which hold health data and physician billing records, we will be able to retrospectively assess the healthcare utilisation, work practices and pregnancy outcomes of women physicians at the population level. Specific outcomes of interest include: (1) rates and timing of pregnancy; (2) pregnancyrelated care and complications; and (3) duration of parental leave and subsequent earnings, each of which will be evaluated with regression methods appropriate to the form of the outcome. We estimate that, at minimum, 5000 women physicians will be eligible for inclusion. Ethics and dissemination This protocol has been approved by the Research Ethics Board at St. Michael's Hospital in Toronto, Ontario, Canada (\#18-248). We will disseminate findings through several peer-reviewed publications, presentations at national and international meetings, and engagement of physicians, residency programmes, department heads and medical societies.

\section{INTRODUCTION}

Despite a marked increase in the number of women entering medicine over the last 50 years, ${ }^{12}$ the challenges associated with becoming pregnant and having children during training or clinical practice have been minimally addressed. ${ }^{3}$ Evidence from qualitative studies and surveys of women physicians
Strengths and limitations of this study

- The observational studies proposed will be the largest to date of women physicians who have experienced pregnancy and childbirth.

- Linkage of the physician cohort to population-based administrative health databases will enable accurate ascertainment of occupational factors such as work intensity that may be associated with pregnancy outcomes.

- Due to the inherent limitations of such databases, we will be unable to account for sociodemographic factors such as relationship status and specific intentions with respect to pregnancy, family planning and work leave practices. We will also be unable to determine the education level or occupation of nonphysician controls.

- This study will be conducted in Ontario, Canada, and may not be generalisable to jurisdictions with major differences in medical training.

raise concerns that pregnancy and motherhood may jeopardise career advancement, reduce job and fellowship opportunities, negatively impact referral patterns and result in resentment from colleagues who may feel hampered with a greater workload..$^{3-10}$ Inconsistent institutional support for pregnant women and parents, and the reality that physician mothers usually bear a disproportionate burden of home and parenting obligations compared with physician fathers, may exacerbate these problems. ${ }^{11-16}$ In part because of these issues, it is thought that women physicians may delay childbearing to more advanced maternal ages, or have fewer or no children more often than nonphysician women in the general population. ${ }^{3}{ }^{17-22}$ However, epidemiological studies investigating such hypotheses are lacking.

Once pregnant, the demands faced by physicians may predispose them to an increased risk of adverse outcomes. Prolonged hours, 
shift/night work and exposure to infectious agents and radiation have been described as potential risk factors for pregnancy complications. ${ }^{23-26}$ Advanced maternal age, due to delayed childbearing, is associated with subfertility as well as increased risks of pregnancy complications including hypertensive disorders, fetal growth restriction, placental abruption, preterm delivery and stillbirth, among others. ${ }^{27}$

Existing studies comparing pregnancy outcomes in physicians and non-physicians are almost exclusively survey based and findings vary widely (table 1). Some studies demonstrate that physicians have increased risks of certain adverse pregnancy outcomes, such as hypertensive disorders and threatened preterm labour, ${ }^{28-33}$ while others find no such relationship. ${ }^{345}$ In the only registrybased study published to date, physician occupation was not associated with preterm labour, low birth weight or perinatal death compared with women with other whitecollar jobs, but differences across specialties, trainee status or work intensity were not investigated. ${ }^{35}$ Since an association between the nature of physicians' work and adverse pregnancy outcomes is biologically plausible, additional high-quality studies are needed.

Women physicians face many challenges after pregnancy, and the literature is limited in this area as well. Although many cross-sectional surveys have identified barriers to obtaining adequate maternity leave and managing clinical loads around delivery and return to work, ${ }^{1236-38}$ few studies have systematically described the practice patterns of physician mothers. ${ }^{16}$ The impact of childbirth and parental leave on the subsequent earnings of women physicians is also unclear. In one survey, over half of physician mothers reported losing US $\$ 10000$ or more in income due to leave. ${ }^{7}$ In other fields, a motherhood earnings penalty beyond the gender pay gap has been noted. ${ }^{39}{ }^{40}$ Although qualitative studies and surveys have underscored a possibly similar phenomenon in physicians, ${ }^{5-7}$ observational research is required.

\section{Specific aims}

In the proposed studies, we will harness unique data resources available in Ontario, Canada, to address unanswered questions in this field. We will first develop a cohort of all physicians who registered to practice in Ontario from 1990 to 2018 by linking physician registration data to existing provincial health administrative data. We will then conduct retrospective analyses within specific subgroups of this larger cohort and a representative sample of non-physicians (figure 1) to address the following objectives:

- Compare reproductive patterns between women physicians and non-physicians, and determine if physician work characteristics are associated with rates of pregnancy.

- Compare maternal outcomes, perinatal outcomes and processes of obstetrical care between women physicians and non-physicians, and determine if physician work characteristics are associated with adverse pregnancy outcomes.

- Describe the pregnancy and postpartum work practices of women physicians who experience childbirth, and determine the impact of childbirth on practice patterns and earnings relative to men physicians and women physicians who do not experience childbirth.

\section{Cohort development}

Existing studies examining issues around pregnancy in physicians are almost entirely self-report surveys with moderate response rates and small sample sizes, susceptible to selection and misclassification bias. We will address this limitation by developing and retrospectively studying a cohort of practising physicians who registered with the College of Physicians and Surgeons of Ontario (CPSO) from 1990 to 2018, linked to existing Ontario population-based administrative databases.

\section{Data sources}

CPSO database

The CPSO is the body that regulates the practice of medicine in Ontario. Physicians are required to be members of the CPSO to practice medicine in the province. The CPSO also has a legislated mandate to continuously improve the quality of care provided by physicians, by maintaining standards of medical practice through peer assessment and remediation.

To do this, the CPSO maintains a database of all physicians who have registered to practice medicine in Ontario. We obtained a dataset of physicians who registered with the CPSO from 1 January 1990 to 26 November 2018 (see online supplemental table 1). This dataset has variables on physicians' registration status, medical school, year of graduation, practice location and specialty, collected at one or two possible time points: (1) the date of physicians' initial registration, and/or (2) the most recent data query.

Physicians of all ages and genders in the CPSO dataset were probabilistically linked to existing provincial administrative databases using physicians' given name, surname, gender and date of birth. Subsets of this larger linked cohort will be used to address each aim (figure 1). The linkage of the CPSO dataset to existing Ontario administrative databases enables assessment of physicians' health service utilisation and health outcomes.

\section{Ontario administrative databases}

All provincial administrative databases (see online supplemental table 2) required to establish the cohorts, exposures, outcomes and covariates specific to each aim are held at ICES, a non-profit research institute authorised to collect and use health data on Ontario residents for the purposes of health system evaluation and improvement. Collection and compilation of health records at ICES is possible because Ontario residents have universal access to physician services and hospital-based care through the Ontario Health Insurance Plan (OHIP). ICES databases 


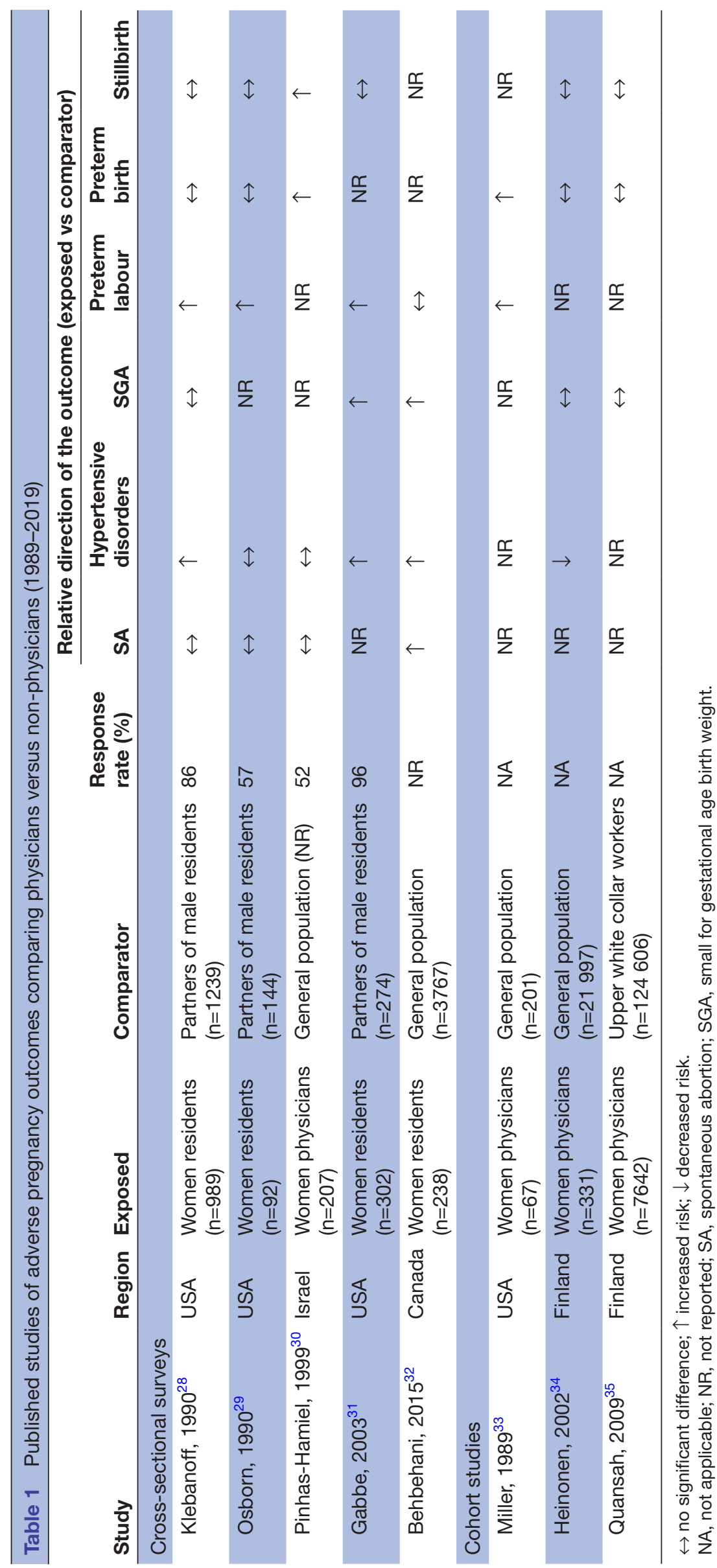

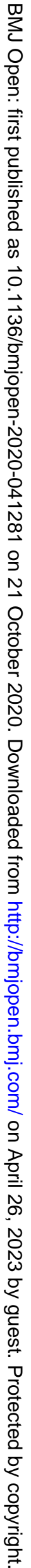




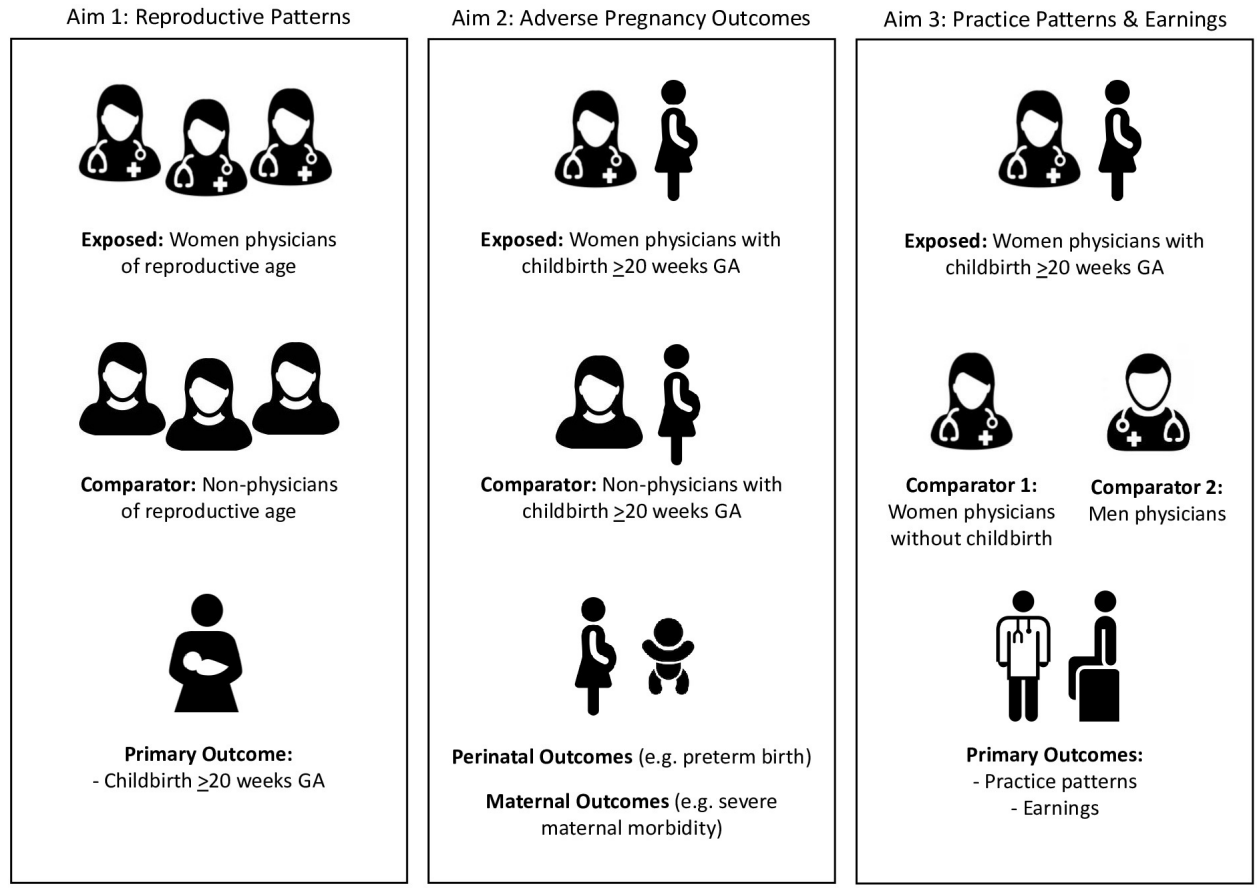

Figure 1 Overview of specific research aims, with study populations (including exposed and comparator groups) and study outcomes. GA, gestational age.

are linked using unique OHIP numbers that are assigned to each individual.

Demographic data will be identified from several ICES databases. Vital statistics and postal code of residence, used to derive rurality and area-level income quintile from Canadian census data, will be obtained from the Registered Persons Database (RPDB). Immigration status will be obtained from the Ontario portion of Immigration, Refugees and Citizenship Canada's Permanent Resident Database. Marginalisation, another area-level measure of socioeconomic status based on residential instability, material deprivation, dependency and ethnic concentration, will be obtained from the Ontario Marginalisation Index.

Comorbidities will be ascertained from the Canadian Institute for Health Information (CIHI) Discharge Abstract Database (DAD), which holds diagnostic/ procedural information on inpatient hospital stays since 1988; the Same Day Surgery (SDS) database, which holds records for same day procedures since 1991; the National Ambulatory Care Reporting System (NACRS), which holds records on emergency department visits since 2000; and the OHIP database, which holds physician billing claims for health services since 1991. Several Ontariospecific registries and ICES-derived cohorts, including the Ontario Cancer Registry, Ontario Diabetes Dataset and Ontario Hypertension Dataset, can also be used identify specific medical conditions.

Childbirths and other recognised pregnancies (eg, spontaneous abortions, ectopic pregnancies) will be identified from the ICES-derived Mother-Baby Dataset (MOMBABY), which links the CIHI records of delivering mothers and their newborns; the Better Outcomes
Registry and Network (BORN), Ontario's perinatal registry including data from fertility clinics, specialised antenatal clinics, hospitals, midwifery practice groups and both prenatal and newborn screening laboratories; as well as the DAD, SDS, OHIP and NACRS databases (see online supplemental tables 2 and 3). Adverse pregnancyrelated and mental health outcomes will be obtained from these same databases as well as the Ontario Mental Health Reporting System database, which holds data on patients in adult designated inpatient mental health beds. Prenatal, antepartum, intrapartum and postpartum health service utilisation, including assisted reproductive technology, will be obtained from the OHIP, DAD/SDS and BORN databases.

The work practices and earnings of Ontario physicians will be obtained from the OHIP database; $95 \%$ of specialists and $50 \%$ of primary care physicians receive their income from fee-for-service (FFS) billings, and all Ontario physicians are required to submit shadow billings for non-FFS services. The frequency and timing of physicians' billing claims for health services and surgical procedures will be used to establish measures of work intensity such as overnight work, and evening and weekend shiftwork, before, during and after pregnancy. Physician earnings will be derived from total OHIP billings. Practice model for family physicians will be obtained from the Client Agency Programme Enrolment database. Specialty, trainee status and practice location, will be obtained from the CPSO dataset and the ICES-derived Physician Database (IPDB), which contains updated yearly information about physicians in Ontario.

Study populations will depend on the aim (figure 1). Aim 1 will include Ontario women of reproductive age. 
Aim 2 will include Ontario women of reproductive age who have had at least one childbirth $\geq 20$ weeks gestational age (GA). In both aims 1 and 2, physician occupation will be the main exposure of interest; we will compare all women physicians (exposed) to a representative sample of non-physicians (comparator). Physicians will be selected from the CPSO dataset. Non-physicians will be selected from the RPDB, and randomly assigned a simulated CPSO registration date based on the distribution of registration dates in physicians.

Aim 3 will include women and men physicians of reproductive age. Childbirth $\geq 20$ weeks GA will be the main exposure of interest; we will compare women physicians who have had at least one childbirth (exposed) to: (1) women physicians who have had no childbirths and (2) men physicians (comparator). Comparator physicians will be randomly assigned a simulated date of childbirth based on the distribution of childbirth dates in women physicians.

\section{Covariates}

We will examine several covariates in physicians and nonphysicians. Demographic factors will include age, year of cohort entry, income quintile and immigration status. Clinical factors will include comorbidities, use of assisted reproductive technology, number of previous livebirths and number of previous recognised pregnancies. We will group comorbidities into Aggregated Diagnosis Groups on the basis of similarity, chronicity, disability and likelihood of requiring specialty care using the Johns Hopkins ACG System. ${ }^{41}$

We will also examine several covariates in physicians only. Trainee status, specialty, practice model, practice location and measures of work intensity (eg, weekend and overnight shifts, time spent operating) will be ascertained according to methodology described below and in previous work. ${ }^{42-44}$

\section{Variable follow-up}

Physicians are a highly mobile population; $34 \%$ of Canadian medical graduates move outside of their home province for residency training, ${ }^{45}$ and $30 \%$ of Canadian physicians in independent practice obtained their medical degree internationally. ${ }^{1}$ We therefore anticipate that some physicians will have lived in Ontario for their entire reproductive lifespans (complete look-back), while others may have left Ontario periodically or arrived for the first time after medical school graduation (incomplete look-back).

Physicians with incomplete look-back prior to their CPSO registration may have insufficient data available to obtain study variables that rely on a historical period, particularly to ascertain previous pregnancies, thus introducing potential for misclassification. For example, a 32-year-old American physician with one prior childbirth moving to Ontario to practice would have no record of that birth in ICES databases. To mitigate this, we will truncate the look-back of non-physicians to mirror that of matched physicians so that they undergo an identical process of ascertaining covariates. This will facilitate appropriate comparison.

\section{Determining transition to independent practice}

The CPSO database contains one variable describing the type of license (eg, postgraduate education, independent practice, etc) held by physicians at the time of their initial registration with the CPSO (see online supplemental table 1). Preliminary analyses demonstrate that $90 \%$ of reproductive-age physicians first registered as residents/ fellows on a postgraduate education license. However, the CPSO database does not hold information on license changes, or when physicians transition from postgraduate education to independent practice.

To mitigate this, we plan to use OHIP data to identify the transition from training to practice. Physicians with a postgraduate education license receive a salary from the provincial Ministry of Health and Long-Term Care, while physicians with an independent practice license receive an income by submitting billings to OHIP. We will use physicians' initiation of billings in OHIP as indicator of their transition from training to practice.

\section{Determining physician specialty}

The CPSO database contains two variables describing the specialty of physicians (see online supplemental table $1)$ : one is collected at initial registration with the CPSO, and the other is collected at the most recent data query. Specialty is not formally assigned until after physicians finish residency training and are certified for practice by either the Royal College of Physicians and Surgeons of Canada or the College of Family Physicians of Canada, despite the fact that they have been working in that specialty for several years.

We will therefore assign specialty from the CPSO database based on information available at the time of either initial registration or the most recent data query. For physicians lacking specialty information, we will use linkages to IPDB and OHIP. If specialty information remains missing after searching all three data sources (CPSO, IPDB, OHIP) and the physician was a recent graduate from medical school $(\geq 2013)$, then such physicians will be categorized as specialty not yet determined.

\section{Use of administrative data sources}

Use of ICES administrative data enables access to a large population-based sample of physicians and nonphysicians, with comprehensive follow-up of all health encounters over the reproductive lifespan. However, ICES administrative data lacks granular variables that would be of interest in this study, such as relationship status and intentions with respect to family planning, and is susceptible to misclassification due to coding errors. We cannot account for unmeasured variables; however, we can mitigate the possibility of information bias. We have purposefully selected main exposures, covariates and outcomes that can be ascertained using established methodology 
and/or Ontario-specific algorithms to ensure accuracy $^{46-54}$; and have used databases that are validated ${ }^{55} 56$ or periodically reabstracted. ${ }^{57}$

\section{Aim 1: compare reproductive patterns in women physicians and non-physicians \\ Rationale and overview}

Numerous survey-based studies suggest that women physicians frequently delay childbearing and subsequently experience a higher rate of infertility compared with the general population. ${ }^{3}{ }^{17-20}$ This has been quantified in only one retrospective cohort study assessing birth trends among Taiwanese female physicians, ${ }^{21}$ which demonstrated that maternal age at delivery was up to 4 years later in physicians than non-physicians. Further studies are needed to characterise the timing and factors impacting pregnancy in physicians.

\section{Analysis plan}

We will retrospectively evaluate reproductive patterns among Ontario women physicians and non-physicians of reproductive age. We will use MOMBABY to ascertain childbirth. Unmatched time-to-event analyses will be performed to compare rates of childbirth between physicians and the general population, and matched or adjusted time-to-event analyses will be used to evaluate the independent association of physician occupation with rates of childbirth. We will also examine secondary outcomes such as number of childbirths and maternal age at childbirth, among physicians and non-physicians.

We also aim to determine whether specific work-related factors faced by physicians impact their reproductive patterns and rates of childbirth. Adjusted time-to-event and Poisson regression models will be constructed in women physicians only to evaluate whether variables such as specialty, trainee status and frequency of overnight work are associated rates of childbirth and other secondary outcomes, respectively.

\section{Aim 2: compare adverse pregnancy outcomes in women physicians and non-physicians \\ Rationale and overview}

It is unclear how work as a physician impacts obstetrical outcomes. A recent systematic review demonstrated that pregnant women who work shifts or longer hours have increased odds of preterm birth and other adverse outcomes, but all included studies were at substantial risk of bias, and only one pertained specifically to physicians. ${ }^{26}$ We will be able to reliably establish work characteristics prior to and during pregnancy from OHIP, and thus provide unique insight into the association between physician occupation and adverse pregnancy outcomes.

\section{Outcomes}

We will retrospectively evaluate adverse pregnancy outcomes among Ontario women physicians and nonphysicians of reproductive age who have experienced at least one childbirth $\geq 20$ weeks GA. All outcomes of interest were chosen for their clinical relevance and established methodology for ascertainment from ICES databases such as MOMBABY, DAD and OHIP, using standard diagnostic and procedural codes ${ }^{46-54}$ (see online supplemental table 3 ).

Perinatal outcomes include: preterm birth (delivery at $<37$ weeks GA); low birth weight; stillbirth; neonatal intensive care unit admission and neonatal death at $<28$ days of life. Maternal outcomes include: severe maternal morbidity (a composite endpoint of potentially lifethreatening complications occurring during the index pregnancy) ${ }^{48}$; maternal death (from 20 weeks GA to $\leq 42$ days post partum); new-onset hypertensive disorders in the index pregnancy; other obstetric (eg, premature rupture of membranes) and non-obstetric complications (eg, peripartum mood disorders); and processes of obstetrical care (eg, antenatal care, labour induction, mode of delivery, epidural).

\section{Analysis plan}

Unmatched logistic regression will be performed to compare each adverse pregnancy outcome specified above between physicians and the general population. Matched or adjusted logistic regression analyses, accounting for demographic and clinical covariates as described above, will be performed to isolate the independent association of physician occupation with adverse pregnancy outcomes. We also aim to determine whether specific work-related factors faced by physicians influence their pregnancy outcomes. Adjusted logistic regression models will be constructed in women physicians only to evaluate whether variables such as specialty, trainee status and overnight work are associated with adverse pregnancy outcomes. For all analyses described, we will also consider use of log-binomial or modified Poisson regression models to determine risk ratios directly.

\section{Aim 3: compare practice patterns and earnings of women physicians experiencing childbirth to non-parent physicians}

Although the challenges faced by both medical trainees and practising physicians in obtaining parental leave have been documented in the literature, ${ }^{5}{ }^{12-38}$ the actual work and leave practices and remuneration of physician mothers are unknown. These data would be of importance to physicians practising in Canada, as the majority are self-employed. We aim to describe the parental leave patterns and earnings of Ontario physicians using a rigorous observational design.

\section{Analysis plan}

We will retrospectively evaluate practice patterns and earnings of men and women physicians in Ontario of reproductive age. We will match women physicians who have had at least one childbirth to women physicians who have had no childbirths, and to men physicians, on their specialty and year of graduation from medical school. Physicians who have delivered will enter the study on their obstetrical delivery date, and physicians who have not delivered will be assigned a corresponding referent date. 
In women physicians who have delivered, we will examine: (1) length of leave, defined by the absence of OHIP billings adjacent to the delivery date and (2) timing of leave, defined in relation to the delivery date. In all physicians, we will examine: (1) work intensity, defined as mentioned previously through evaluation of measures such as overnight call practices and operating time; (4) earnings, as defined by OHIP billings.

We will compare earnings across three distinct periods: (1) prepregnancy, (2) peripartum and (3) postpregnancy. We will first perform a within-patient analysis pertaining to delivering women physicians only, in order to assess how their earnings vary with pregnancy and childbirth. Earnings from all three time periods will be compared using regression methods for cost data (eg, Poisson, negative binomial, gamma models); the specific model will be determined based on the distribution of earnings for the cohort.

We will then perform a comparative analysis of (1) delivering women physicians to non-delivering women physicians and (2) delivering women physicians to men physicians. Earnings from the prepregnancy and postpregnancy time periods, or dummy time periods in controls, will again be evaluated with appropriate regression methods for cost data.

\section{Sample size and power}

The CPSO dataset should have adequate power for all proposed analyses. To demonstrate this, we have calculated the power of our study to find differences in adverse pregnancy outcomes, specifically preterm birth, between women physicians and non-physicians (specific aim 2). Preterm birth is a major determinant of neonatal morbidity/mortality, and has significant long-term health consequences. Even a small increased risk of preterm birth would be of importance to women physicians.

If a conservative 5000 physicians have at least one pregnancy during the study period, are compared with at least 25000 non-physicians, and we assume a baseline preterm birth rate of 7.7 per 100 births $^{58}$ and an alpha of 0.05 , we will have $80 \%$ power to detect a relative risk of 1.16 or greater, and $90 \%$ power to detect a relative risk of 1.19 or greater.

\section{Patient and public involvement}

The public were not involved in the design of this study. The proposed research questions aim to address issues of importance to physician health; the study team accordingly includes women physicians and physician parents.

\section{Significance}

The linkage of physician information to population-based data on pregnancy presents a unique opportunity to evaluate physicians' reproductive patterns and perinatal health outcomes in a manner that addresses the limitations of previous studies. Ontario's FFS system allows accurate ascertainment of physician work intensity and other work-related factors that may affect rates of reproduction and adverse pregnancy outcomes.
This work is needed; reproductive patterns and childbearing have not been rigorously studied in physicians, despite many barriers to pregnancy and risk factors for adverse outcomes inherent in their work. We will determine if physicians are at increased risk of adverse pregnancy outcomes compared with the general population, and clarify whether this risk is mediated by age or other occupational hazards. Understanding issues around pregnancy and leave, which may affect up to half of the physician workforce at some point during their careers, also has implications for the functioning of the healthcare system.

\section{Ethics and dissemination}

This protocol was approved by the Research Ethics Board at St. Michael's Hospital (\#18-248) and by the ICES Privacy \& Legal Office. ICES is a prescribed entity under section 45 of Ontario's Personal Health Information Protection Act. Section 45 authorises ICES to collect personal health information without consent for analyses related to the evaluation of, allocation of resources to, or planning for all or part of the health system. In accordance with ICES policy, we will suppress all cells with $<6$ individuals to prevent reidentification. All research outputs related to this work will undergo a reidentification risk assessment prior to submission.

Translation of the findings of our study into practices and policies will require engagement of physicians, physician leaders and organisational bodies. The team of researchers includes clinician investigators in obstetrics, surgery, medicine and psychiatry who will provide important contextual information to the dissemination of our findings. We will engage bodies such as the Society of Obstetricians and Gynaecologists of Canada, the Canadian Medical Association, and residency programmes and department heads.

We anticipate that our findings will be presented at local and national conferences, and result in several peerreviewed publications. All manuscripts will adhere to the Strengthening the Reporting of Observational Studies in Epidemiology guidelines (online supplemental table 4). Our findings should impact physicians, physicians-intraining, medical educators, residency programme directors, department chairs, and hospitals and organisations where physicians work.

\section{Author affiliations}

${ }^{1}$ Department of Obstetrics \& Gynaecology, University of Toronto, Toronto, Ontario, Canada

${ }^{2}$ Li Ka Shing Knowledge Institute, St. Michael's Hospital, Toronto, Ontario, Canada ${ }^{3}$ Melbourne School of Population and Global Heath, University of Melbourne, Melbourne, Victoria, Australia

${ }^{4}$ ICES (Formerly the Institute for Clinical Evaluative Sciences), Toronto, Ontario, Canada

${ }^{5}$ Division of Biostatistics, Dalla Lana School of Public Health, University of Toronto, Toronto, Ontario, Canada

${ }^{6}$ Department of Medicine, St. Michael's Hospital, Toronto, Ontario, Canada

${ }^{7}$ Department of Medicine, London Health Sciences Centre, London, Ontario, Canada ${ }^{8}$ Department of Psychiatry, Women's College Hospital, Toronto, Ontario, Canada ${ }^{9}$ Department of Psychiatry, Women's College Research Institute, Women's College Hospital, Toronto, Ontario, Canada 
${ }^{10}$ Department of Obstetrics \& Gynaecology, St. Michael's Hospital/Unity Health Toronto, Toronto, Ontario, Canada

\section{Twitter Maria C Cusimano @mccusimano}

Acknowledgements The authors thank Dr Peter Tanuseputro, Dr Manish Sood and Emily Rhodes, Research Assistant in Clinical Epidemiology at the Ottawa Hospital Research Institute, for their assistance with data acquisition.

Contributors All authors (MCC, NNB, RS, JGR, AG, EM, SV and ANS) contributed to the design of this study. NNB, AG and ANS participated in data acquisition. MCC, NNB, RS, JGR, EM and ANS developed the analytic plan. MCC, NNB and ANS obtained ethics approval for this work. MCC prepared the first draft of the manuscript. All authors contributed to and approved the final version of the manuscript.

Funding This study will be conducted with grant funding from Physicians' Services Incorporated (PSI) Foundation. This study is also supported by ICES, which is funded by an annual grant from the Ontario Ministry of Health and Long-Term Care (MOHLTC). The opinions, results, analytical plans and conclusions reported in this paper are those of the authors and are independent of the funding sources. No endorsement by ICES or the Ontario MOHLTC is intended or should be inferred. Dr Maria Cusimano is supported by the American College of Surgeons Resident Research Scholarship and the Canadian Institutes of Health Research (CIHR) Vanier Canada Graduate Scholarship.

Competing interests None declared.

Patient consent for publication Not required.

Provenance and peer review Not commissioned; externally peer reviewed.

Open access This is an open access article distributed in accordance with the Creative Commons Attribution Non Commercial (CC BY-NC 4.0) license, which permits others to distribute, remix, adapt, build upon this work non-commercially, and license their derivative works on different terms, provided the original work is properly cited, appropriate credit is given, any changes made indicated, and the use is non-commercial. See: http://creativecommons.org/licenses/by-nc/4.0/.

ORCID iD

Maria C Cusimano http://orcid.org/0000-0002-1661-4846

\section{REFERENCES}

1 ClHI. Physicians in Canada: Canadian Institute for health information, 2018. Available: https://www.cihi.ca/en/physicians-in-canada

2 AAMC. Physician specialty data report: association of American medical colleges, 2018. Available: https://www.aamc.org/system/ files/2019-08/2018executivesummary.pdf [Accessed October 7, 2020].

3 Turner PL, Lumpkins K, Gabre J, et al. Pregnancy among women surgeons: trends over time. Arch Surg 2012;147:474-9.

4 Tamburrino MB, Evans CL, Campbell NB, et al. Physician pregnancy: male and female colleagues' attitudes. J Am Med Womens Assoc 1992;47:82-4.

5 Halley MC, Rustagi AS, Torres JS, et al. Physician mothers' experience of workplace discrimination: a qualitative analysis. $B M$ 2018;363:k4926.

6 Adesoye T, Mangurian C, Choo EK, et al. Perceived discrimination experienced by physician mothers and desired workplace changes: a cross-sectional survey. JAMA Intern Med 2017;177:1033-6.

7 Scully RE, Davids JS, Melnitchouk N. Impact of procedural specialty on maternity leave and career satisfaction among female physicians. Ann Surg 2017;266:210-7.

8 Krause ML, Elrashidi MY, Halvorsen AJ, et al. Impact of pregnancy and gender on internal medicine resident evaluations: a retrospective cohort study. J Gen Intern Med 2017;32:648-53.

9 Kin C, Yang R, Desai P, et al. Female trainees believe that having children will negatively impact their careers: results of a quantitative survey of trainees at an academic medical center. BMC Med Educ 2018;18:260

10 Willett LL, Wellons MF, Hartig JR, et al. Do women residents delay childbearing due to perceived career threats? Acad Med 2010;85:640-6.

11 Humphries LS, Lyon S, Garza R, et al. Parental leave policies in graduate medical education: a systematic review. Am J Surg 2017;214:634-9.

12 Phillips SP, Richardson B, Lent B. Medical faculty's views and experiences of parental leave: a collaborative study by the gender issues Committee, Council of Ontario faculties of medicine. J Am Med Womens Assoc 2000;55:23-6.

13 MacVane CZ, Fix ML, Strout TD, et al. Congratulations, you're pregnant! now about your shifts: the state of maternity leave attitudes and culture in EM. West J Emerg Med 2017;18:800-10.

14 Hutchinson AM, Anderson NS, Gochnour GL, et al. Pregnancy and childbirth during family medicine residency training. Fam Med 2011;43:160-5.

15 Jolly S, Griffith KA, DeCastro R, et al. Gender differences in time spent on parenting and domestic responsibilities by high-achieving young physician-researchers. Ann Intern Med 2014;160:344353-53.

16 Ly DP, Seabury SA, Jena AB. Hours worked among US dual physician couples with children, 2000 to 2015. JAMA Intern Med 2017;177:1524-5.

17 Aghajanova L, Hoffman J, Mok-Lin E, et al. Obstetrics and gynecology residency and fertility needs. Reprod $\mathrm{Sci}$ 2017;24:428-34.

18 Troppmann KM, Palis BE, Goodnight JE, et al. Women surgeons in the new millennium. Arch Surg 2009;144:635-42.

19 Stentz NC, Griffith KA, Perkins E, et al. Fertility and childbearing among American female physicians. J Womens Health 2016;25:1059-65.

20 Bering J, Pflibsen L, Eno C, et al. Deferred personal life decisions of women physicians. J Womens Health 2018;27:584-9.

21 Wang Y-J, Chiang S-C, Chen T-J, et al. Birth trends among female physicians in Taiwan: a nationwide survey from 1996 to 2013. Int J Environ Res Public Health 2017;14:746.

22 Souza JP, Tunçalp Ö, Vogel JP, et al. Obstetric transition: the pathway towards ending preventable maternal deaths. BJOG 2014;121 Suppl $1: 1-4$

23 Katz VL, Miller NH, Bowes WA, Katz VL. Pregnancy complications of physicians. West J Med 1988;149:704-7.

24 Palmer KT, Bonzini M, Harris EC, et al. Work activities and risk of prematurity, low birth weight and pre-eclampsia: an updated review with meta-analysis. Occup Environ Med 2013;70:213-22.

25 Stocker LJ, Macklon NS, Cheong YC, et al. Influence of shift work on early reproductive outcomes: a systematic review and meta-analysis. Obstet Gynecol 2014;124:99-110.

26 Cai C, Vandermeer B, Khurana R, et al. The impact of occupational shift work and working hours during pregnancy on health outcomes: a systematic review and meta-analysis. Am J Obstet Gynecol 2019;221:563-76.

27 Lean SC, Derricott H, Jones RL, et al. Advanced maternal age and adverse pregnancy outcomes: a systematic review and metaanalysis. PLoS One 2017;12:e0186287.

28 Klebanoff MA, Shiono PH, Rhoads GG. Outcomes of pregnancy in a national sample of resident physicians. N Engl J Med 1990;323:1040-5.

29 Osborn LM, Harris DL, Reading JC, et al. Outcome of pregnancies experienced during residency. J Fam Pract 1990;31:618-22.

30 Pinhas-Hamiel O, Rotstein Z, Achiron A, et al. Pregnancy during residency--an Israeli survey of women physicians. Health Care Women Int 1999;20:63-70.

31 Gabbe SG, Morgan MA, Power ML, et al. Duty hours and pregnancy outcome among residents in obstetrics and gynecology. Obstet Gynecol 2003;102:948-51.

32 Behbehani S, Tulandi T. Obstetrical complications in pregnant medical and surgical residents. J Obstet Gynaecol Can 2015;37:25-31.

33 Miller NH, Katz VL, Cefalo RC. Pregnancies among physicians. A historical cohort study. J Reprod Med 1989;34:790-6.

34 Heinonen S, Saarikoski S. Reproductive risk factors, pregnancy characteristics and obstetric outcome in female doctors. BJOG 2002;109:261-4.

35 Quansah R, Gissler M, Jaakkola JJK. Work as a physician and adverse pregnancy outcomes: a Finnish nationwide populationbased registry study. Eur J Epidemiol 2009;24:531-6.

36 Rangel EL, Castillo-Angeles M, Changala M, et al. Perspectives of pregnancy and motherhood among general surgery residents: a qualitative analysis. Am J Surg 2018;216:754-9.

37 Rangel EL, Smink DS, Castillo-Angeles M, et al. Pregnancy and motherhood during surgical training. JAMA Surg 2018;153:644-52.

38 Mohan H, Ali O, Gokani V, et al. Surgical trainees' experience of pregnancy, maternity and paternity leave: a cross-sectional study. Postgrad Med J 2019;95:552-7.

39 Budig MJ, England P. The wage penalty for motherhood. Am Sociol Rev 2001;66:204-25

40 Budig M, Hodges MJ. Differences in disadvantage: variation in the motherhood penalty across white women's earnings distribution. Am Sociol Rev 2010;75:705-28. 
41 Austin PC, van Walraven C, Wodchis WP, et al. Using the Johns Hopkins aggregated diagnosis groups (ADGS) to predict mortality in a general adult population cohort in Ontario, Canada. Med Care 2011;49:932-9.

42 Govindarajan A, Urbach DR, Kumar M, et al. Outcomes of daytime procedures performed by attending surgeons after night work. $N$ Engl J Med 2015;373:845-53.

43 Redelmeier DA, Thiruchelvam D, Daneman N. Introducing a methodology for estimating duration of surgery in health services research. J Clin Epidemiol 2008;61:882-9.

44 Dossa F, Simpson AN, Sutradhar R, et al. Sex-Based disparities in the hourly Earnings of surgeons in the fee-for-service system in Ontario, Canada. JAMA Surg 2019;154:1134.

45 CaRMS. R-1 Data and Reports Ottawa. Ontario: Canadian resident matching service, 2019. Available: https://www.carms.ca/datareports/r1-data-reports/

46 Ray JG, Vermeulen MJ, Schull MJ, et al. Cardiovascular health after maternal placental syndromes (CHAMPS): population-based retrospective cohort study. Lancet 2005;366:1797-803.

47 Joseph KS, Liu S, Rouleau J, et al. Severe maternal morbidity in Canada, 2003 to 2007: surveillance using routine hospitalization data and ICD-10CA codes. J Obstet Gynaecol Can 2010;32:837-46

48 Ray JG, Park AL, Dzakpasu S, et al. Prevalence of severe maternal morbidity and factors associated with maternal mortality in Ontario, Canada. JAMA Netw Open 2018;1:e184571.

49 Garg AX, Nevis IF, McArthur E, et al. Gestational hypertension and preeclampsia in living kidney donors. N Engl J Med 2015;372:124-33.
50 Wanigaratne S, Shakya Y, Gagnon AJ, et al. Refugee maternal and perinatal health in Ontario, Canada: a retrospective population-based study. BMJ Open 2018;8:e018979.

51 Ray JG, Park AL, Fell DB. Mortality in infants affected by preterm birth and severe Small-for-Gestational age birth weight. Pediatrics 2017;140:e20171881.

52 Bartsch E, Park AL, Jairam J, et al. Concomitant preterm birth and severe small-for-gestational age birth weight among infants of immigrant mothers in Ontario originating from the Philippines and East Asia: a population-based study. BMJ Open 2017;7:e015386.

53 Polachek IS, Fung K, Vigod SN. First lifetime psychiatric admission in the postpartum period: a population-based comparison to women with prior psychiatric admission. Gen Hosp Psychiatry 2016;40:25-32.

54 Barker LC, Kurdyak P, Fung K, et al. Postpartum psychiatric emergency visits: a nested case-control study. Arch Womens Ment Health 2016;19:1019-27.

55 Tu K, Campbell NR, Chen Z-L, et al. Accuracy of administrative databases in identifying patients with hypertension. Open Med 2007;1:e18-26.

56 Lipscombe LL, Hwee J, Webster L, et al. Identifying diabetes cases from administrative data: a population-based validation study. BMC Health Serv Res 2018;18:316.

57 Juurlink D, Preyra C, Croxford R, et al. Canadian Institute for health information discharge Abstract database: a validation study. Toronto: Institute for Clinical Evaluative Sciences, 2006.

58 Health Fact Sheets. Trends in Canadian births: 1993 to 2013: statistics Canada. Available: https://www150.statcan.gc.ca/n1/pub/ 82-625-x/2016001/article/14673-eng.htm 